\title{
Overexpressing transcriptional regulator in Chaetomium globosum activates a silent biosynthetic pathway: evaluation of shanorellin biosynthesis
}

\author{
Yuta Tsunematsu ${ }^{1}$, Suguru Ichinoseki ${ }^{1}$, Takehito Nakazawa ${ }^{1}$, Noriyasu Ishikawa ${ }^{1}$, Hiroshi Noguchi ${ }^{1}$, \\ Kinya Hotta ${ }^{2}$ and Kenji Watanabe ${ }^{1}$
}

The Journal of Antibiotics (2012) 65, 377-380; doi:10.1038/ja.2012.34; published online 9 May 2012

Keywords: biosynthesis; fungi; PKS; silent gene cluster; transcriptional regulator

In recent years, many secondary metabolite gene clusters, including polyketide biosynthetic genes, have been uncovered through fungal genome sequencing. Although approximately 33 polyketide biosynthetic genes can be identified in the genome of a fungus Chaetomium globosum, only 11 fungal polyketide scaffolds ${ }^{1}$ can be isolated from cultures grown under typical growth conditions. ${ }^{2}$ Transcription factors (TFs) located within a gene cluster often determine whether its respective secondary metabolites can be biosynthesized, and it is this class of regulators that is responsible for the low expression level of biosynthetic genes and less-than-desirable yield of the corresponding natural products. ${ }^{3,4}$ Here, we explored the feasibility of activating a silent gene cluster through overexpression of a TF encoded in a putative polyketide biosynthetic gene cluster in C. globosum for which a stably propagating expression vector is unavailable.

For the current study, we focused on a putative polyketide biosynthetic gene cluster from C. globosum. DNA sequence analysis of this 25-kilobase (kb)-long cluster (Figure 1a) revealed the presence of seven genes, $\operatorname{cgs} A-G$ (Table 1), that appeared to be responsible for a polyketide biosynthesis. In order to activate this silent gene cluster involving a large polyketide synthase (PKS) gene $(>7.1 \mathrm{~kb})$, a recombinant strain capable of overexpressing the transcriptional factor $\operatorname{cgs} G$ was constructed. Instead of activating the expression of the endogenous copy of $\operatorname{cgs} G$ within the $\operatorname{cgs}$ cluster, we chose to introduce a separate copy of $\operatorname{cgs} G$ under the control of a constitutively active promoter to achieve overexpression of the TF in C. globosum to avoid potential repression of the transcription of $c g s G$ located within the cluster by some other regulation mechanism. For the overexpression of this regulator, which is highly homologous to a known GAL4-like DNA-binding protein, we chose to use pBluescript SK as the base for the delivery vector for transforming C. globosum with a second copy of constitutively expressing $\operatorname{cgs} G$. For the construction of the delivery vector, PCR was performed with the C. globosum genomic
DNA as a template to amplify the $\beta$-tubulin $(t u b)$ promoter and terminator. Similarly, a $h p h$ gene encoding for a hygromycin phosphotransferase that confers hygromycin resistance in C. globosum was amplified from pPHT1 plasmid ${ }^{5}$ as a selectable marker. These three fragments, $t u b$ promoter, $h p h$ and tub terminator, were assembled into a single fragment by overlap extension PCR. The resulting $h p h$ expression cassette was inserted into pBluescript using Geneart Seamless Cloning and Assembly Kit (Life Technologies, Grand Island, NY, USA) to yield the delivery vector, pKW3202. Also, the actA promoter, which is responsible for the transcription of the actin gene, was isolated from the C. globosum chromosomal DNA and inserted into pKW3202 to prepare pKW13000. Subsequently, the 1.4-kb DNA fragment containing the coding and terminator region of $\operatorname{cgs} G$ was inserted immediately downstream of the actA promoter in pKW13000 to yield pKW12203, a hygromycin resistance-conferring vector capable of overexpressing $\operatorname{cgs} G$ (Figure $1 \mathrm{~b}$ ). Transformation was carried out as described elsewhere ${ }^{6}$ with the following modification: the recipient C. globosum strain was initially cultivated in MYG medium containing $0.8 \mathrm{M}$ sorbitol and $200 \mu \mathrm{g} \mathrm{ml}^{-1}$ hygromycin. The plasmid pKW12203 was linearized by $\mathrm{XbaI}$ and transformed into C. globosum. Approximately 50 transformants were grown on MYG agar plates supplemented with $200 \mu \mathrm{g} \mathrm{ml}^{-1}$ hygromycin. The selection confirmed the integration of pKW12203 carrying $\operatorname{cgs} G$ into the genome of the transformant. This transformed C. globosum strain was termed CGKW1.

Next, we examined whether transcription of $\operatorname{cgs} A$ encoding for a PKS can be activated when the transcriptional regulator CgsG is overexpressed. Total RNA $(1 \mu \mathrm{g})$ isolated from CGKW1 was subjected to reverse transcription-PCR for $c g s A$ cDNA synthesis with a random hexamer primer (Applied Biosystems, Life Technologies). The cDNA formation was detected by agarose gel electrophoresis (Figure $1 \mathrm{~b}, \operatorname{cgs} \mathrm{A}$ ) following PCR amplification of cDNA using the following primer set: CHGG8793coreFw, 5'-TCCATGGATCCCCAGCAGAGG-3' and 

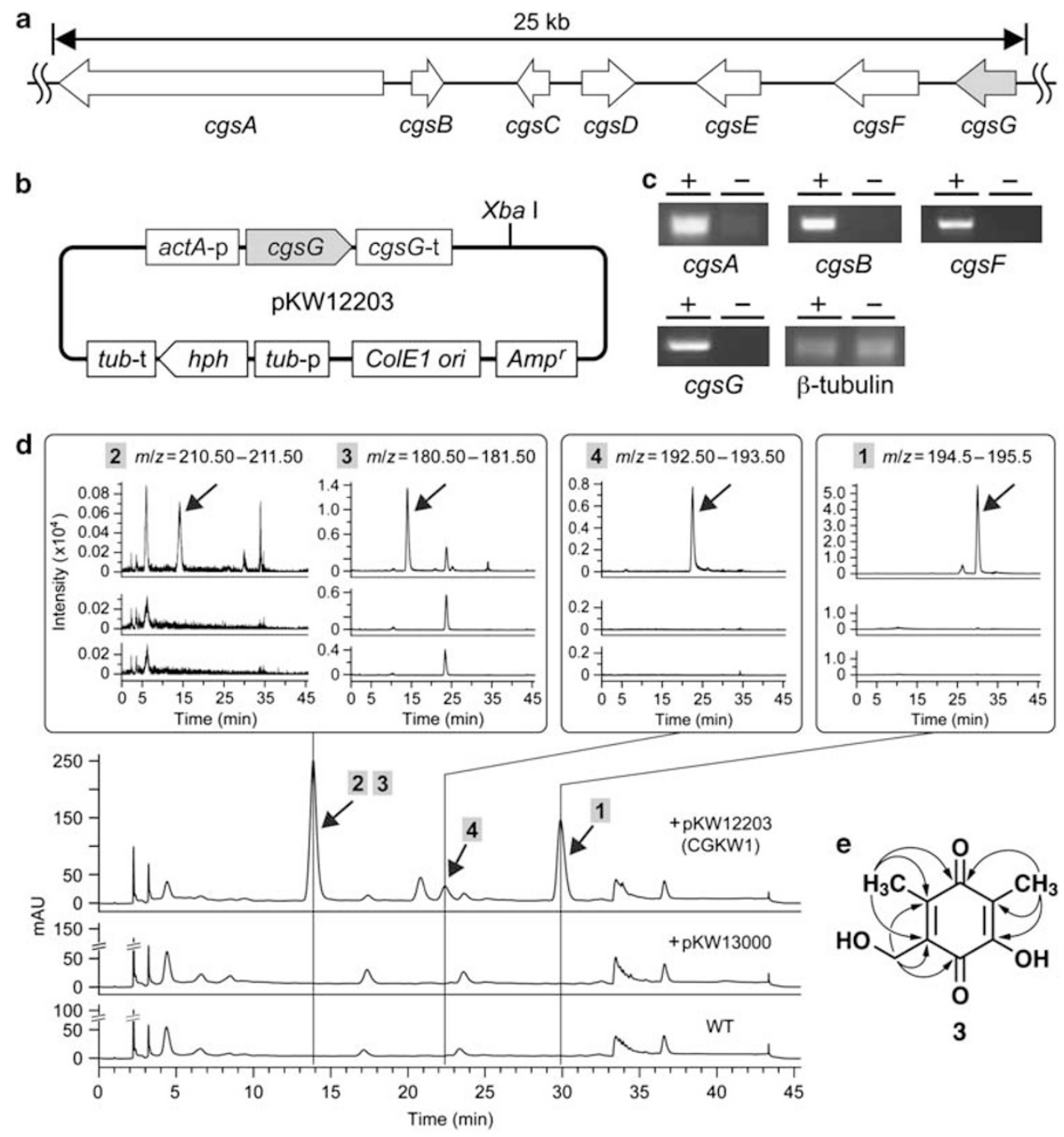

$\mathbf{f}$

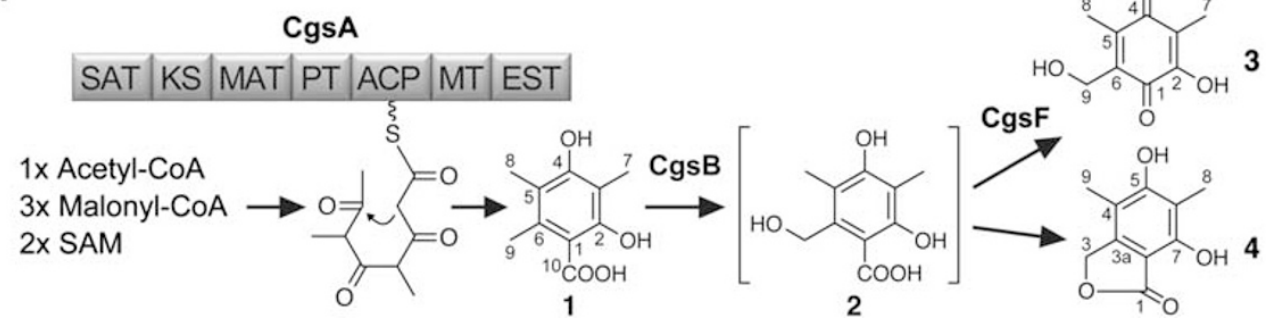

Figure 1 Characterization of the silent cgs gene cluster, activation of transcription from the cluster, and the products biosynthesized by the associated enzymes. (a) Organization of the cgs gene cluster involved in the biosynthesis of 3. (b) Map of the plasmid pKW12203 carrying the hygromycin phosphotransferase $(h p h)$ gene under the control of a $\beta$-tubulin promoter (tub-p) and a $\beta$-tubulin terminator (tub-t), along with the transcription regulator $\operatorname{cgs} G$ gene under the control of an actin promoter (actA-p) and the original $\operatorname{cgs} G$ terminator (cgsG-t). (c) Reverse transcription-PCR analysis of the effect of overexpressing the transcriptional regulator $\operatorname{cgs} G$ on the expression of three other cgs genes, $\operatorname{cgs} A$, $\operatorname{cgs} B$ and $\operatorname{cgs} F$ in $C$. globosum. Expression of the $\beta$-tubulin gene was used as a positive control. -: C. globosum transformed with an empty plasmid pKW13000 carrying no cgs gene; +: CGKW1, a C. globosum strain overexpressing the transcriptional regulator gene cgsG introduced by pKW12203. (d) Sets of three HPLC traces from the LC-MS analysis of the metabolic extracts from the wild-type and recombinant strains of $C$. globosum for determining the effect of overexpressing the transcriptional regulator CgsG on activating the silent cgs biosynthetic gene cluster. In each set of three chromatograms, the top one is from $C$. globosum strain CGKW1, the middle one is from C. globosum carrying pKW13000, and the bottom one is from the wild-type C. globosum. All traces were monitored at $254 \mathrm{~nm}$. Boxed chromatograms are the extracted ion chromatograms for the range of $\mathrm{m} / \mathrm{z}$ covering the compounds 1-4. (e) Analysis of the HMBC data for $\mathbf{3}$. (f) Proposed biosynthetic pathway of $\mathbf{3}$ from a polyketide product $\mathbf{1}$ by the activated cgs gene cluster. Predicted domain organization of the iterative PKS CgsA is also shown. The domains, depicted as shaded boxes, are: SAT, starter unit; ACP transacylase; KS, ketosynthase; MAT, malonyl-CoA acyltransferase; PT, product template; ACP, acyl carrier protein; MT, methyltransferase; EST, lipase-like esterase. 
Table 1 Deduced functions of the open reading frames ORFs in the cgs biosynthetic gene cluster from $C$. globosum

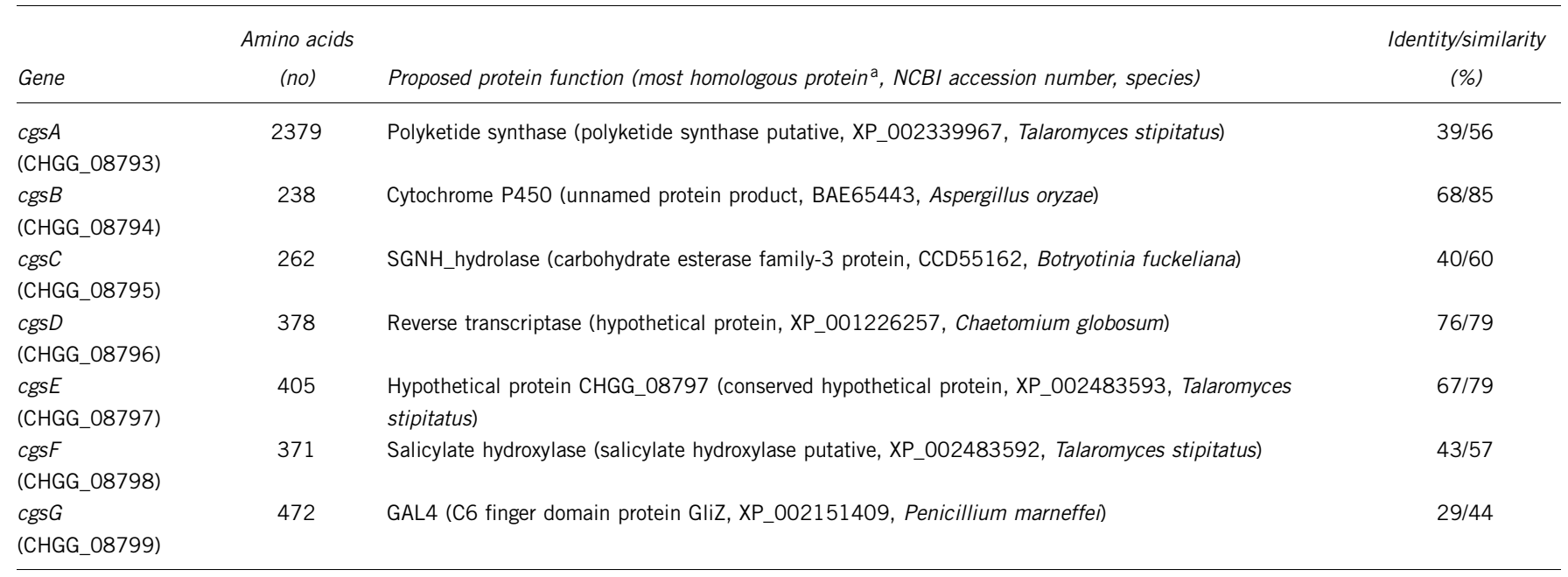

aDeduced functions of the ORFs identified within the cgs biosynthetic gene cluster were based on the percentage sequence similarity/identity to known proteins as determined by Protein BLAST search against the NCBI non-redundant database.

CHGG8793coreRv, 5'-ACCCGTCCCATGAGCCTCCAC-3'. As a negative control, the absence of transcription of $\operatorname{cgs} A$ was confirmed in $C$. globosum transformed with pKW12203 lacking the $\operatorname{cgs} G$ insertion (Figure 1c, WT). Transcription of the $\beta$-tubulin gene was used as a positive control to confirm that general gene transcription remained similar in both the wild-type and the CGKW1 strain (Figure 1c, $\beta$ tubulin). Similar investigations revealed successful activation of $\operatorname{cgs} B$ and $c g s F$ genes upon overexpression of an extraneous copy of $\operatorname{cgs} G$ (Figure $1 c, c g s B$ and $c g s F$ ).

To isolate and characterize the polyketide compound(s) biosynthesized by the cgs cluster, CGKW1 was cultivated on an oatmeal agar for 7 days at $30^{\circ} \mathrm{C}$. For the control samples, the wild-type C. globosum and C. globosum carrying a blank plasmid pKW13000 were also cultured under the same condition. Reversed-phase LC-MS analysis of the crude organic extract of the culture broth revealed the presence of several nonpolar metabolites that were present only in the CGKW1 culture broth (Figure 1d). For a full characterization of those compounds, we cultivated the strain on MYG liquid medium on a large scale and purified the compounds using flash column chromatography and HPLC when necessary. NMR data, including ${ }^{1} \mathrm{H}$ and ${ }^{13} \mathrm{C}$ spectra, as well as HMBC analysis (Figure 1e), allowed determination of the chemical structure of the compound eluted at around 13.9 min to be shanorellin (3). ${ }^{7}$ Another compound, 2,4-dihydroxy3,5,6-trimethylbenzoic acid (1) ${ }^{8}$ a presumed intermediate compound for the biosynthesis of $\mathbf{3}$ (Figure 1f), was also isolated from CGKW1. Furthermore, a phthalide, 5,7-dihydroxy-4,6-dimethylisobenzofuran-1(3H)-one (4), ${ }^{9}$ was isolated from the culture of CGKW1. The results of the spectrometric analyses of these compounds are given below.

Compound 1: Yellowish white powder; ${ }^{1} \mathrm{H}$ NMR (acetone- $d_{6}$, $500 \mathrm{MHz}): \delta=2.50(3 \mathrm{H}, \mathrm{s}), 2.15(3 \mathrm{H}, \mathrm{s}), 2.11(3 \mathrm{H}, \mathrm{s}) ;{ }^{13} \mathrm{C} \mathrm{NMR}$ (acetone- $\left.d_{6}, 125 \mathrm{MHz}\right): \delta=174.9(\mathrm{C}-10), 161.6$ (C-2), 159.0 (C-4), 138.6 (C-6), 116.4 (C-5), 108.8 (C-3), 105.7 (C-1), 18.9 (C-9), 12.3 (C-8), 8.7 (C-7); ESI-MS: $m / z 195$ [M-H] ${ }^{-}$; HRESIMS: $m / z 195.0654$ $\left([\mathrm{M}-\mathrm{H}]^{-}\right.$, calcd. for $\left.\mathrm{C}_{10} \mathrm{H}_{11} \mathrm{O}_{4}, 195.0663\right)$.

Compound 3: Orange crystal; ${ }^{1} \mathrm{H}$ NMR $\left(\mathrm{CDCl}_{3}, 500 \mathrm{MHz}\right)$ : $\delta=4.58(2 \mathrm{H}, \mathrm{s}, \mathrm{H}-9), 2.14(3 \mathrm{H}, \mathrm{s}, \mathrm{H}-8), 1.94(3 \mathrm{H}, \mathrm{s}, \mathrm{H}-7) ;{ }^{13} \mathrm{C}$ NMR $\left(\mathrm{CDCl}_{3}, 125 \mathrm{MHz}\right): \delta=187.8(\mathrm{C}-4), 184.1(\mathrm{C}-1), 150.9(\mathrm{C}-2)$, 145.4 (C-5), 136.8 (C-6), 118.0 (C-3), 56.7 (C-9), 12.5 (C-8),
8.4 (C-7); ESI-MS: $m / z 181[\mathrm{M}-\mathrm{H}]^{-}$; HRESIMS: $m / z 181.0503$ $\left([\mathrm{M}-\mathrm{H}]^{-}\right.$, calcd. for $\left.\mathrm{C}_{9} \mathrm{H}_{9} \mathrm{O}_{4}, 181.0506\right)$.

Compound 4: Yellowish white powder; ${ }^{1} \mathrm{H}$ NMR (acetone- $d_{6}$, $500 \mathrm{MHz}): \delta=8.42(1 \mathrm{H}$, brs, OH-5), $8.02(1 \mathrm{H}$, brs, OH-7), 5.26 $(2 \mathrm{H}, \mathrm{s}, \mathrm{H}-3), 2.14(3 \mathrm{H}, \mathrm{s}, \mathrm{H}-8), 2.12(3 \mathrm{H}, \mathrm{s}, \mathrm{H}-9) ;{ }^{13} \mathrm{C} \mathrm{NMR}$ (acetone- $\left.d_{6}, 125 \mathrm{MHz}\right): \delta=173.2(\mathrm{C}-1), 161.3(\mathrm{C}-5), 154.1$ (C-7), 145.3 (C-3a), 111.4 (C-6), 110.6 (C-4), 103.3 (C-7a), 70.3 (C-3), 11.3 (C-9), 8.3 (C-8); ESI-MS: $m / z 195[\mathrm{M}+\mathrm{H}]^{+}$; HRESIMS: $m / z$ $195.0652\left([\mathrm{M}+\mathrm{H}]^{+}\right.$, calcd. for $\left.\mathrm{C}_{10} \mathrm{H}_{11} \mathrm{O}_{4}, 195.0652\right)$.

Our result revealed that the $c g s$ gene cluster is responsible for the biosynthesis of compound 3. This cluster contains $\operatorname{cgs} A$ that encodes a $255-\mathrm{kDa}$ iterative PKS with seven domains as ascertained by in silico sequence analysis: starter unit ACP transacylase, ketosynthase, malonyl-CoA acyltransferase, product template, acyl carrier protein, methyltransferase and lipase-like esterase (Figure 1f). The analysis also identifies that CgsA can be categorized as a non-reducing PKS owing to its lack of domains capable of catalyzing a reduction and/or dehydration reaction. ${ }^{10}$ Considering the relatively high sequence similarity between CgsA and the 5-methylorsellinic acid-synthesizing $\mathrm{MpaC}$ (37.6\% identity, $52.7 \%$ similarity), it is highly plausible that CgsA synthesizes the benzoic acid core structure of $1^{8}$ from one molecule of acetyl-CoA and three molecules of malonyl-CoA. Then the methyltransferase domain in CgsA can transfer two methyl groups $^{7}$ from $S$-adenosyl-L-methionine to $\mathrm{C} 7$ and C8 to generate $\mathbf{1}$.

BLAST $^{11}$ and FFAS03 ${ }^{12}$ sequence analyses of other enzymes encoded in the cgs cluster suggest that the polyketide backbone is modified by two more enzymes to afford $\mathbf{3}$ as the final product (Figure 1f). The first step is likely catalyzed by CgsB, which is predicted to be a cytochrome P450. CgsB could perform hydroxylation of the C9 methyl substituent in the PKS product 1 to afford the presumed intermediate 2. Although 2 was not isolated from the culture directly, the LC-MS analysis identified that 2 co-elutes with the final product 3 as a minor constituent (Figure 1d). Also, a phthalide 4, which can be formed by spontaneous lactonization of $2,{ }^{13}$ was identified by the LC-MS analysis, providing further support for the involvement of $\mathbf{2}$ as an intermediate of the pathway. Then, the final step for the formation of $\mathbf{3}$ can be carried out by CgsF, which is highly homologous to salicylate hydroxylase. Salicylate hydroxylase belongs to a flavin-dependent mono-oxygenase family 
and is responsible for the decarboxylative hydroxylation of salicylate to form catechol with a reduction of an enzyme-bound FAD by NADH under aerobic conditions. ${ }^{14,15}$ Analogously, CgsF can perform a decarboxylative hydroxylation to convert a dihydroxybenzoic acid 2 to a hydroxyhydroquinone product, which can be readily oxidized into the final hydroxyquinone product, shanorellin 3.

In this study, we successfully activated a silent polyketide biosynthetic gene cluster involving an iterative PKS present in C. globosum by constitutively overexpressing an extraneous copy of a TF associated with the cluster. Activation of the silent gene cluster was confirmed by reverse transcription-PCR. A strict correlation between the expression of the otherwise silent $c g s$ genes and the production of several polyketide products strongly indicates that the identified polyketide products are indeed the products of the Cgs biosynthetic enzymes. This was the first successful activation of polyketide biosynthesis from a silent gene cluster by overexpressing its associated TF in C. globosum. Our results showed that CgsA is an iterative PKS capable of synthesizing 2,4-dihydroxy-3,5,6-trimethylbenzoic acid (1). Furthermore, we were able to identify two tailoring enzymes, a cytochrome $\mathrm{P} 450 \mathrm{CgsB}$ and a salicylate hydroxylase homolog CgsF in the gene cluster. Through this work, we were able to establish experimentally that the silent $c g s$ cluster is responsible for shanorellin (3) biosynthesis. In addition, we were able to propose the shanorellin biosynthetic pathway involving CgsB hydroxylating the C6 methyl group of the initial polyketide product 1 to generate 2 , followed by CgsF converting 2 into shanorellin (3) via a decarboxylative hydroxylation. Our result clearly demonstrates that our approach of plasmid-based overexpression of TF is a practical approach toward mining for new natural products that can be biosynthesized by fungi. Furthermore, our methodology can help expand our knowledge of novel enzymes and their associated metabolic pathways that would facilitate our efforts toward engineering biosynthetic pathways for production of analogs with useful bioactivity.

\section{ACKNOWLEDGEMENTS}

We would like to express our appreciation to the financial support from Japan Society for the Promotion of Science (JSPS) through the 'Funding Program for Next Generation World-Leading Researchers', initiated by the Council for
Science and Technology Policy (No. LS103) (KW) and by Industrial Technology Research Grant Program in 2009 (No. 09C46001a) from the New Energy and Industrial Technology Development Organization (NEDO) of Japan (KW). This work was also supported in part by the Mochida memorial foundation (Japan) for medical and pharmaceutical research (KW), by Takeda Science Foundation (KW), by Noda Institute for Scientific Research Grants (KW) and by a Grant-in-Aid for Research Activity start-up from JSPS (TN). Postdoctoral fellowships to YT and TN from JSPS are gratefully acknowledged.

1 Scherlach, K., Boettger, D., Remme, N. \& Hertweck, C. The chemistry and biology of cytochalasans. Nat. Prod. Rep. 27, 869-886 (2010).

2 Brakhage, A. A. \& Schroeckh, V. Fungal secondary metabolites - strategies to activate silent gene clusters. Fungal. Genet. Biol. 48, 15-22 (2010).

3 Flaherty, J. E. \& Payne, G. A. Overexpression of aflR leads to upregulation of pathway gene transcription and Increased aflatoxin production in Aspergillus flavus. Appl. Environ. Microbiol. 63, 3995-4000 (1997)

4 Bergmann, S. et al. Genomics-driven discovery of PKS-NRPS hybrid metabolites from Aspergillus nidulans. Nat. Chem. Biol. 3, 213-217 (2007).

5 Cummings, W. J., Celerin, M., Crodian, J., Brunick, L. K. \& Zolan, M. E. Insertiona mutagenesis in Coprinus cinereus: use of a dominant selectable marker to generate tagged, sporulation-defective mutants. Curr. Genet. 36, 371-382 (1999).

6 Shibuya, I. et al. Molecular cloning of the glucoamylase gene of Aspergillus shirousami and its expression in Aspergillus oryzae. Agric. Biol. Chem. 54, 1905-1914 (1990).

7 Wat, C., McInnes, A. G., Smith, D. G. \& Vining, L. C. Use of ${ }^{13} \mathrm{C}$ in biosynthetic studies Location of isotope from labeled acetate and formate in shanorellin by ${ }^{13} \mathrm{C}$ nuclear magnetic resonance spectrometry. Can. J. Biochem. 50, 620-624 (1972).

8 Hirota, A., Morimitsu, Y. \& Hojo, H. New antioxidative indophenol-reducing phenol compounds isolated from the Mortierella sp. Fungus. Biosci. Biotech. Biochem. 61, 647-650 (1997).

9 Achenbach, H., Mühlenfeld, A. \& Brillinger, G. U. Phthalide und chromanole aus Aspergillus duricaulis. Liebigs Ann. Chem. 808, 1596-1628 (1985).

10 Chiang, Y. M., Oakley, B. R., Keller, N. \& Wang, C. C. Unraveling polyketide synthesis in members of the genus Aspergillus. Appl. Microbiol. Biotechnol. 86, 1719-1736 (2010).

11 Johnson, M., Zaretskaya, I., Raytselis, Y., Merezhuk, Y., McGinnis, S. \& Madden, T. L. NCBI BLAST: A better web interface. Nucleic Acids Res. 36, W5-W9 (2008) (http://blast.ncbi.nlm.nih.gov/Blast.cgi)

12 Jaroszewski, L., Rychlewski, L., Li, Z., Li, W. \& Godzik, A. FFAS03: a server for profile-profile sequence alignments. Nucleic Acids Res. 33, W284-W288 (2005) (http://ffas.burnham.org/ffas-cgi/cgi/document.pl).

13 Ichihara, A., Adachi, K., Hosokawa, K. \& Takeda, Y. Enzymatic hydroxylation of aromatic carboxylic acids. J. Biol. Chem. 237, 2296-2302 (1962).

14 Katagiri, $M$ et al. Salicylate hydroxylase, a monooxygenase requiring flavin adenine dinucleotide. II. The mechanism of salicylate hydroxylation to catechol. J. Biol. Chem. 240, 3414-3417 (1965).

15 Suzuki, K., Gomi, T. \& Itagaki, E. Intermediate and mechanism of hydroxylation of o-iodophenol by salicylate hydroxylase. J. Biochem. 109, 791-797 (1991). 